\title{
Perinatal outcomes and related factors: social class differences within and between geographical areas
}

\author{
DIANA ELBOURNE, ${ }^{1}$ COLIN PRITCHARD ${ }^{2}$ AND MARJORIE DAUNCEY 3 \\ From the National Perinatal Epidemiology Unit, ${ }^{1}$ Radcliffe Infirmary, Oxford, OX2 6HE; Medical Research \\ Council Medical Sociology Unit, ${ }^{2}$ Glasgow*; and Department of Medical Computing and Statistics, ${ }^{3}$ University of \\ Wales College of Medicine, Cardiff
}

SUMMARY This paper makes use of the opportunity provided by comparable obstetric data bases to examine area and social class variations in perinatal outcome and associated factors in areas smaller than those usually reported. Analyses are based on singleton births to primiparous residents in the catchment areas of the Aberdeen Maternity and Neonatal Database $(n=4948)$ and the Cardiff Births Survey $(n=11893)$ between 1976 and 1981. The factors considered relate to the obstetric population (height, age, and smoking), obstetric practice (induction and assisted delivery), and perinatal outcome (curtailed gestation, low birthweight, and perinatal death). Our analysis confirms the existence of both area and social class differences and suggests that, except in the case of teenage pregnancy and smoking, the association observed between those factors and area and social class are largely independent of each other.

There exists a large body of research ${ }^{1}$ demonstrating the variety of relationships between the circumstances in which people live and aspects of human reproduction. Most of this has shown that the chance of adverse perinatal outcomes and their associated risk factors are consistently higher for babies born to women in working class compared with middle class families, and are also higher for babies born to mothers whose usual place of residence is in the north or west compared with the south or east of Britain.

The authors of the Decennial Supplement for the years 1970-72 considered both class and regional mortality together. They concluded that "although social class does explain some of the differences in infant mortality between the regions, marked differences remain unaccounted for".

The broad objectives of this paper are to explore their conclusion further, focussing instead on perinatal mortality. Variations in the incidence of factors associated with perinatal death ${ }^{1}$ are also considered. For each of these variables (death and risk factors) a series of four interrelated questions are posed. The first pair of questions relates to comparisons between geographical areas and asks:

1 Is there a difference in the overall incidence of the variable in question between the areas considered?

2 Are differences repeated throughout the social class distribution?

*Present address: Social, paediatric and obstetric research unit, Glasgow
The second pair of questions relates to comparisons between social classes and asks:

3 Is there a relationship between the variable in question and social class?

4 Is this relationship similar for the different areas being compared?

\section{Materials and methods}

\section{DATA BASES}

The National Perinatal Epidemiology Unit Aberdeen/ Cardiff/Oxford Collaborative Project was created to provide a common framework for research between three well established perinatal data bases. The present study is based on the two data bases in Aberdeen and in Cardiff.

The Aberdeen Maternity and Neonatal Data Bank $(A M N D B)^{3}$ was established in 1948 and in recent years covers all births to residents of Aberdeen City and District.

The Cardiff Births Survey (CBS) 4 was established nearly 20 years later, in 1965 . From 1974 it has covered births to residents of an area which, until the 1982 National Health Service reorganisation, was the South Glamorgan Area Health Authority, an obstetric population nearly three times the size of AMNDB.

The study period is 1976 to 1981 . This is mainly because of the availability of comparable data over this time. The obstetric populations are described in terms of parity and multiplicity (table 1). Because of 
the small numbers of multiple births, and problems of their data interpretation-particularly for birthweight - further analysis is restricted to singleton births. To avoid the possibility of double-counting, multiparous women are also excluded. Hence all subsequent analysis is based on singleton births to primiparous residents of the Aberdeen and Cardiff data base catchment areas between 1976 and 1981 .

VAR I A B L ES

In both the AMNDB and the CBS, social class of married women is based on the Registrar General's 1970 classification derived from the occupation of the woman's husband. Classes I and II, and IV and V are aggregated to provide large enough subgroups for the analysis. Classes III manual and non-manual are also aggregated as it is not possible to separate them in the CBS. Women who were not married at the time of delivery are considered as a separate category. In addition to the categories of classes I to $\mathrm{V}$ and "not married', there is a residual group including women married to students and members of the armed forces, others and those for whom there was inadequate information to permit classification. The social class distributions in the AMNDB and the CBS are shown in table 2. They clearly differ, the AMNDB having proportionally more women described as classes IV and $\mathrm{V}$ than the CBS.

All the variables that are used for the following analyses have been selected because previous research has demonstrated an association with social class ${ }^{1}$ and because they have been collected comparably in the two data bases. They can be divided into three broad and interrelated groups.

Obstetric population Each of the variables, short stature, smoking, and extremes of maternal age, have been consistently associated with both social class and perinatal outcome. In this study, short stature refers to

Table 1 Obstetric population 1976-81

\begin{tabular}{lrr}
\hline & AMNDB & $C B S$ \\
\hline Singleton & & \\
pregnancies & & \\
Primiparae & 4989 & 11893 \\
Multiparae & 5525 & 17130 \\
Unknown parity & 0 & 25 \\
All & 10514 & 29048 \\
& & \\
Multiple & & \\
pregnancies & 48 & 105 \\
Primiparae & 56 & 194 \\
Multiparae & 0 & 1 \\
Unknown parity & 104 & 300 \\
All & 10618 & 29348 \\
Total & & \\
\hline
\end{tabular}

Table 2 Social class distribution, 1976-81

\begin{tabular}{|c|c|c|c|c|}
\hline & \multicolumn{2}{|c|}{$A M N D B$} & \multicolumn{2}{|l|}{$C B S$} \\
\hline & No. & $\%$ & No. & $\%$ \\
\hline Class I and II & 907 & $18 \cdot 2$ & 2296 & $19 \cdot 3$ \\
\hline III & 1853 & $37 \cdot 1$ & 4755 & 40.0 \\
\hline IV and $V$ & 1127 & $22 \cdot 6$ & 1539 & $12 \cdot 9$ \\
\hline Not married & 910 & $18 \cdot 2$ & 2441 & $20 \cdot 5$ \\
\hline Miscellaneous & 192 & 3.9 & 862 & $7 \cdot 3$ \\
\hline Total & 4989 & 100 & 11893 & 100 \\
\hline
\end{tabular}

women whose height is $154 \mathrm{~cm}$ or less. Smoking during pregnancy is treated as a dichotomous (yes/no) variable. Extremes of maternal age are defined as ages of 19 years or less (teenage) and 30 or more.

Obstetric practice Area variations in obstetric practice may relate both to the population served and to particular local policies. Two aspects of practice which may be considered as a response to perceptions of increased risk are discussed-the induction of labour (including elective caesarean section) and assisted delivery (operative and instrumental).

Pregnancy outcome Both area and social class differences in pregnancy outcome have been widely reported. Outcomes considered here are preterm delivery ( $<37$ completed weeks' gestation); low birthweight $(<2500 \mathrm{~g})$; and perinantal death (stillbirths and first week deaths).

\section{STATISTICAL METHODS}

In order to make the requisite comparisons, the tables show the estimated relative risks ${ }^{5}$ of selected adverse factors associated with social class and place of residence. In general, an estimated relative risk greater than unity indicates a worse situation in lower compared with higher social classes, or in the AMNDB compared with the CBS. Because some of these comparisons involve relatively small sample sizes, the $95 \%$ confidence intervals ${ }^{6}$ are also quoted. Therefore a difference is considered statistically significant at the $5 \%$ level only if this interval does not include unity.

\section{Results}

AREA COMPARISONS

Obstetric populations (fig 1 and table 3)

Overall, comparisons between the areas show a higher proportion of women of short stature (fig la) and of women who smoke cigarettes in pregnancy (fig ld) in the AMNDB than in the CBS, but a lower proportion of women aged over 29 years (fig 1c). There was no statistically significant difference in the rate of teenage pregnancies (fig lb). 
Table 3 Area comparisons: obstetric population

\begin{tabular}{|c|c|c|c|c|c|c|c|c|c|c|c|c|}
\hline \multirow[b]{3}{*}{$\mathbf{N}$} & \multicolumn{12}{|c|}{ Social class } \\
\hline & \multicolumn{2}{|l|}{ I \& II } & \multicolumn{2}{|l|}{ III } & \multicolumn{2}{|c|}{ IV \& V } & \multicolumn{2}{|c|}{ Unmarried } & \multicolumn{2}{|c|}{ Miscellaneous } & \multicolumn{2}{|l|}{ All } \\
\hline & $\begin{array}{l}\text { A } \\
907\end{array}$ & $\begin{array}{l}C \\
2296\end{array}$ & $\begin{array}{l}\text { A } \\
1853\end{array}$ & $\begin{array}{l}C \\
4755\end{array}$ & $\begin{array}{l}\mathrm{A} \\
1127\end{array}$ & $\begin{array}{l}C \\
1539\end{array}$ & $\begin{array}{l}A \\
910\end{array}$ & $\begin{array}{l}C \\
2441\end{array}$ & $\begin{array}{l}A \\
192\end{array}$ & $\begin{array}{l}C \\
862\end{array}$ & $\begin{array}{l}A \\
4989\end{array}$ & $\begin{array}{l}\text { C } \\
11893\end{array}$ \\
\hline $\begin{array}{l}\leqslant 154 \mathrm{~cm}, \\
\% \\
\text { RR } \\
95 \% \text { CI }\end{array}$ & \multicolumn{2}{|c|}{$\begin{array}{c}1.31^{*} \\
1.07-1.59\end{array}$} & \multicolumn{2}{|c|}{$\begin{array}{c}1 \cdot 31^{*} \\
1 \cdot 16-1.47\end{array}$} & \multicolumn{2}{|c|}{$\begin{array}{c}1.26^{*} \\
1.09-1.46\end{array}$} & \multicolumn{2}{|c|}{$\begin{array}{c}1.29^{*} \\
1.11-1.50\end{array}$} & \multicolumn{2}{|c|}{$\begin{array}{c}1.44^{*} \\
1.07-1.93\end{array}$} & \multicolumn{2}{|c|}{$\begin{array}{c}1.38^{*} \\
1.21-1.39\end{array}$} \\
\hline $\begin{array}{l}<20 \text { years } \\
\% \\
\text { RR } \\
95 \% \text { CI }\end{array}$ & \multicolumn{2}{|c|}{$\begin{array}{c}2.47^{*} \\
1.65-3.71\end{array}$} & \multicolumn{2}{|c|}{$\begin{array}{c}1.18^{*} \\
1.03-1.34\end{array}$} & 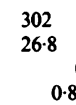 & $\begin{array}{l}457 \\
29 \cdot 7 \\
90 \\
-1.02\end{array}$ & $\begin{array}{c}0.97 \\
0.91-1.04\end{array}$ & $\begin{array}{l}1440 \\
59.0 \\
97 \\
-1.04\end{array}$ & 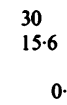 & $\begin{array}{c}0.69^{*} \\
0.49-0.98\end{array}$ & \multicolumn{2}{|c|}{$\begin{array}{c}1.01 \\
0.96-1.07\end{array}$} \\
\hline $\begin{array}{l}>29 \text { years } \\
\% \\
\text { RR } \\
95 \% \mathrm{CI}\end{array}$ & \multicolumn{2}{|c|}{$\begin{array}{c}0.74^{*} \\
0.63-0.86\end{array}$} & \multicolumn{2}{|c|}{$\begin{array}{c}0.65^{*} \\
0.54-0.79\end{array}$} & $\begin{array}{l}57 \\
5 \cdot 1 \\
0.6\end{array}$ & $\begin{array}{c}88 \\
5.7 \\
-1.22\end{array}$ & $\begin{array}{r}24 \\
2.6 \\
0\end{array}$ & $\begin{array}{c}\quad 03 \\
.02 \\
-1.63\end{array}$ & 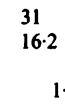 & $\begin{array}{c}69 \\
8 \cdot 0 \\
02^{*} \\
-3.00\end{array}$ & $\begin{array}{c}0.78^{*} \\
0.70-0.87\end{array}$ & $\begin{array}{l}1230 \\
10 \cdot 3 \\
78^{*} \\
0.87\end{array}$ \\
\hline $\begin{array}{l}\text { Smokers } \\
\% \\
\text { RR } \\
95 \% \text { CI }\end{array}$ & \multicolumn{2}{|c|}{$\begin{array}{c}1.52^{*} \\
1.33-1.75\end{array}$} & $\begin{array}{r}841 \\
45 \cdot 4 \\
1.2\end{array}$ & $\begin{array}{c}1639 \\
34 \cdot 5 \\
-32 * \\
3-1 \cdot 41\end{array}$ & $\begin{array}{r}624 \\
55.4 \\
1.0\end{array}$ & $\begin{array}{c}727 \\
47 \cdot 2 \\
17 * \\
-1 \cdot 26\end{array}$ & $\begin{array}{r}620 \\
68 \cdot 1 \\
1\end{array}$ & $\begin{array}{c}1459 \\
59.8 \\
14^{*} \\
-1.21\end{array}$ & $\begin{array}{l}\begin{array}{l}81 \\
42 \cdot 2 \\
1\end{array} \\
1\end{array}$ & $\begin{array}{c}250 \\
29.0 \\
46^{*} \\
3-1.79\end{array}$ & \multicolumn{2}{|c|}{$\begin{array}{c}1 \cdot 25^{*} \\
1 \cdot 21-1 \cdot 30\end{array}$} \\
\hline
\end{tabular}

$* p<0.05$

For both height and smoking these area differences were observed within all of the social class categories. The pattern is less clear for maternal age. Among those for whom it was possible to assign a social class, the area difference in the likelihood of being an older mother is maintained, but this difference is not evident for unmarried mothers and is reversed for the 'miscellaneous' category. The only exception to the lack of area difference in teenage pregnancies is their markedly higher proportion in social classes I, II and III in the AMNDB.

Obstetric practice (fig 2 and table 4)

There was a threefold relative risk of a nonspontaneous onset of labour in the AMNDB compared with the CBS (fig 2a), although there were no significant differences in the rate of assisted delivery between the areas (fig $2 b$ ). These area comparisons were substantially the same throughout the social class distribution. The only exception was area differences in the induction rate among the unmarried category. This was due to the relatively high proportion of labours with spontaneous onset in the CBS, rather than a high rate of induction among this group in the AMNDB.

Outcome (fig 3 and table 5)

There was a significantly higher preterm delivery rate in the AMNDB compared with the CBS (fig 3a) but no significant differences between the areas in terms of

Table 4 Area comparisons: obstetric practice

\begin{tabular}{|c|c|c|c|c|c|c|c|c|c|c|c|c|}
\hline \multirow[b]{3}{*}{$\mathbf{N}$} & \multicolumn{12}{|c|}{ Social class } \\
\hline & \multicolumn{2}{|l|}{ I \& II } & \multicolumn{2}{|l|}{ III } & \multicolumn{2}{|c|}{ IV \& V } & \multicolumn{2}{|c|}{ Unmarried } & \multicolumn{2}{|c|}{ Miscellaneous } & \multicolumn{2}{|l|}{ All } \\
\hline & $\begin{array}{l}\text { A } \\
907\end{array}$ & $\begin{array}{l}\text { C } \\
2296\end{array}$ & $\begin{array}{l}A \\
1853\end{array}$ & $\begin{array}{l}C \\
4755\end{array}$ & $\begin{array}{l}A \\
1127\end{array}$ & $\begin{array}{l}\mathrm{C} \\
1539\end{array}$ & $\begin{array}{l}A \\
910\end{array}$ & $\begin{array}{l}C \\
2441\end{array}$ & $\begin{array}{l}A \\
192\end{array}$ & $\begin{array}{l}C \\
862\end{array}$ & $\begin{array}{l}A \\
4989\end{array}$ & $\begin{array}{l}\mathrm{C} \\
11893\end{array}$ \\
\hline $\begin{array}{l}\text { Induction } \\
\%\end{array}$ & $\begin{array}{l}388 \\
42 \cdot 8\end{array}$ & $\begin{array}{l}357 \\
15.6\end{array}$ & $\begin{array}{l}795 \\
42 \cdot 9\end{array}$ & $\begin{array}{l}644 \\
13 \cdot 5\end{array}$ & $\begin{array}{l}450 \\
39 \cdot 2\end{array}$ & $\begin{array}{l}207 \\
13 \cdot 5\end{array}$ & $\begin{array}{l}325 \\
35 \cdot 7\end{array}$ & $\begin{array}{r}197 \\
8 \cdot 1\end{array}$ & $\begin{array}{l}73 \\
38 \cdot 0\end{array}$ & $\begin{array}{l}114 \\
13 \cdot 2\end{array}$ & $\begin{array}{r}2031 \\
40.7\end{array}$ & $\begin{array}{r}1519 \\
12 \cdot 8\end{array}$ \\
\hline $\begin{array}{l}\text { RR } \\
\mathbf{9 5 \%} \text { CI }\end{array}$ & \multicolumn{2}{|c|}{$\begin{array}{c}2 \cdot 75^{*} \\
2 \cdot 44-3 \cdot 10\end{array}$} & \multicolumn{2}{|c|}{$\begin{array}{c}3 \cdot 17^{*} \\
2 \cdot 90-3 \cdot 46\end{array}$} & \multicolumn{2}{|c|}{$\begin{array}{c}2.97 * \\
2 \cdot 59-3 \cdot 40\end{array}$} & \multicolumn{2}{|c|}{$\begin{array}{c}4 \cdot 43^{*} \\
3 \cdot 81-5 \cdot 13\end{array}$} & \multicolumn{2}{|c|}{$\begin{array}{c}2 \cdot 88^{*} \\
2 \cdot 23-3 \cdot 71\end{array}$} & \multicolumn{2}{|c|}{$\begin{array}{c}3.17^{*} \\
3.00-3.36\end{array}$} \\
\hline \multirow{2}{*}{$\begin{array}{l}\text { Assisted } \\
\text { delivery } \\
\% \\
\text { RR } \\
95 \% \text { CI }\end{array}$} & $\begin{array}{l}424 \\
46 \cdot 7\end{array}$ & $\begin{array}{c}1137 \\
49 \cdot 5\end{array}$ & $\begin{array}{l}839 \\
45 \cdot 3\end{array}$ & $\begin{array}{c}2241 \\
47 \cdot 1\end{array}$ & $\begin{array}{l}471 \\
41 \cdot 8\end{array}$ & $\begin{array}{l}696 \\
45 \cdot 2\end{array}$ & $\begin{array}{l}346 \\
38 \cdot 0\end{array}$ & $\begin{array}{c}888 \\
36 \cdot 8\end{array}$ & $\begin{array}{l}84 \\
43 \cdot 8\end{array}$ & $\begin{array}{l}376 \\
43.6\end{array}$ & $\begin{array}{r}2164 \\
43.4\end{array}$ & $\begin{array}{l}5338 \\
44.9\end{array}$ \\
\hline & \multicolumn{2}{|c|}{$\begin{array}{c}0.94 \\
0.87-1.02\end{array}$} & \multicolumn{2}{|c|}{$\begin{array}{c}0.96 \\
0.91-1.02\end{array}$} & \multicolumn{2}{|c|}{$\begin{array}{c}0.92 \\
0.85-1.01\end{array}$} & \multicolumn{2}{|c|}{$\begin{array}{c}1.05 \\
0.95-1.15\end{array}$} & \multicolumn{2}{|c|}{$\begin{array}{c}1.00 \\
0.84-1 \cdot 20\end{array}$} & \multicolumn{2}{|c|}{$\begin{array}{c}0.97 \\
0.93-1.00\end{array}$} \\
\hline
\end{tabular}




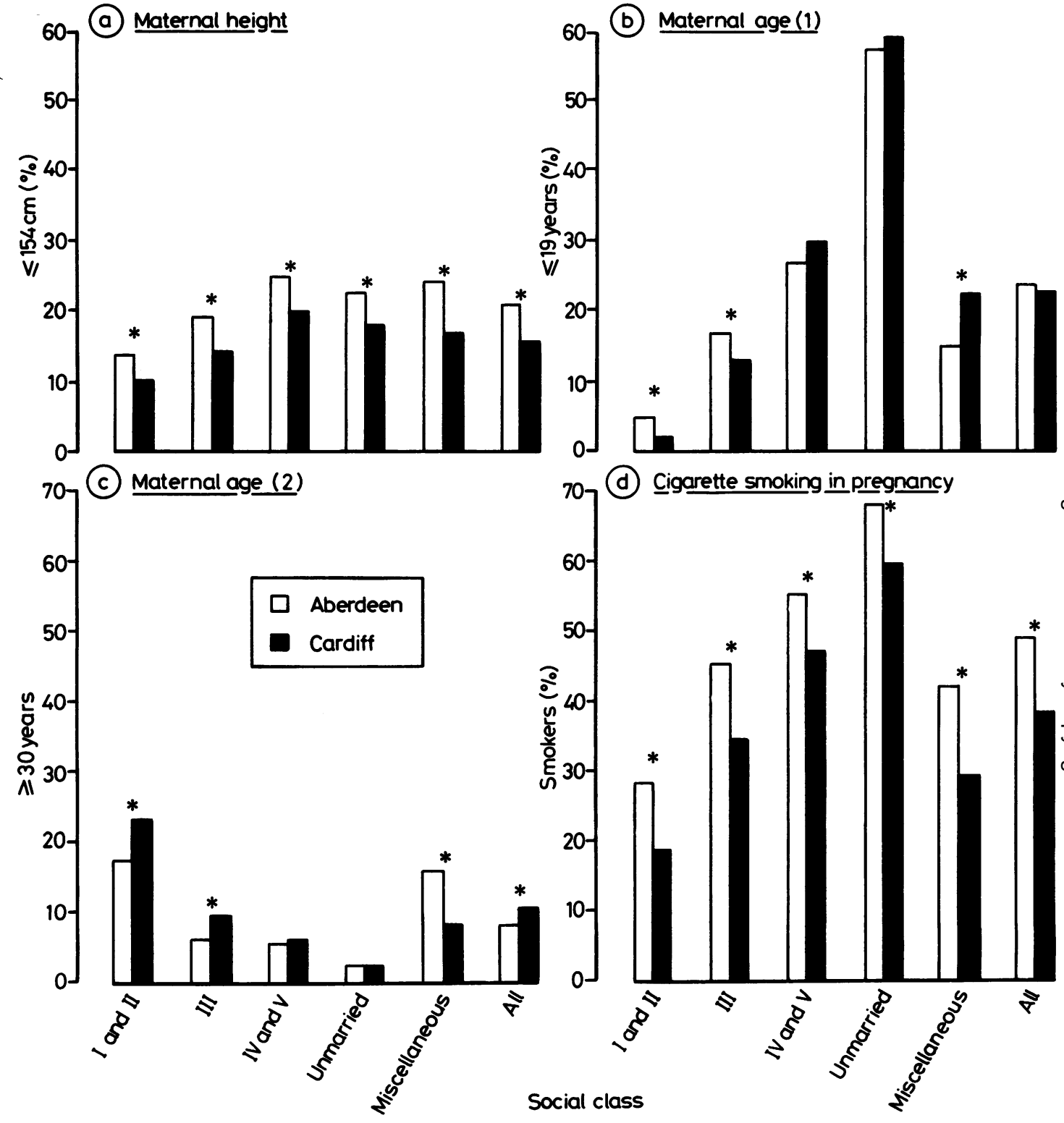

Fig 1

rates of low birthweight or perinatal death. Again, these area patterns were reflected throughout the social class distribution.

In summary, for the majority of the variables considered there are area differences. These area differences are in general maintained throughout the social class distribution.
SOCIAL CLASS COMPARISONS

Obstetric population (fig 1 and table 6)

All these variables exhibit the 'usual' class relationship, ie, women in social classes IV and $\mathrm{V}$ tend to be shorter (fig la), more likely to smoke cigarettes (fig ld), and be younger (fig $1 \mathrm{~b}$ and fig $1 \mathrm{c}$ ) than women in social classes I and II. There are area differences in 

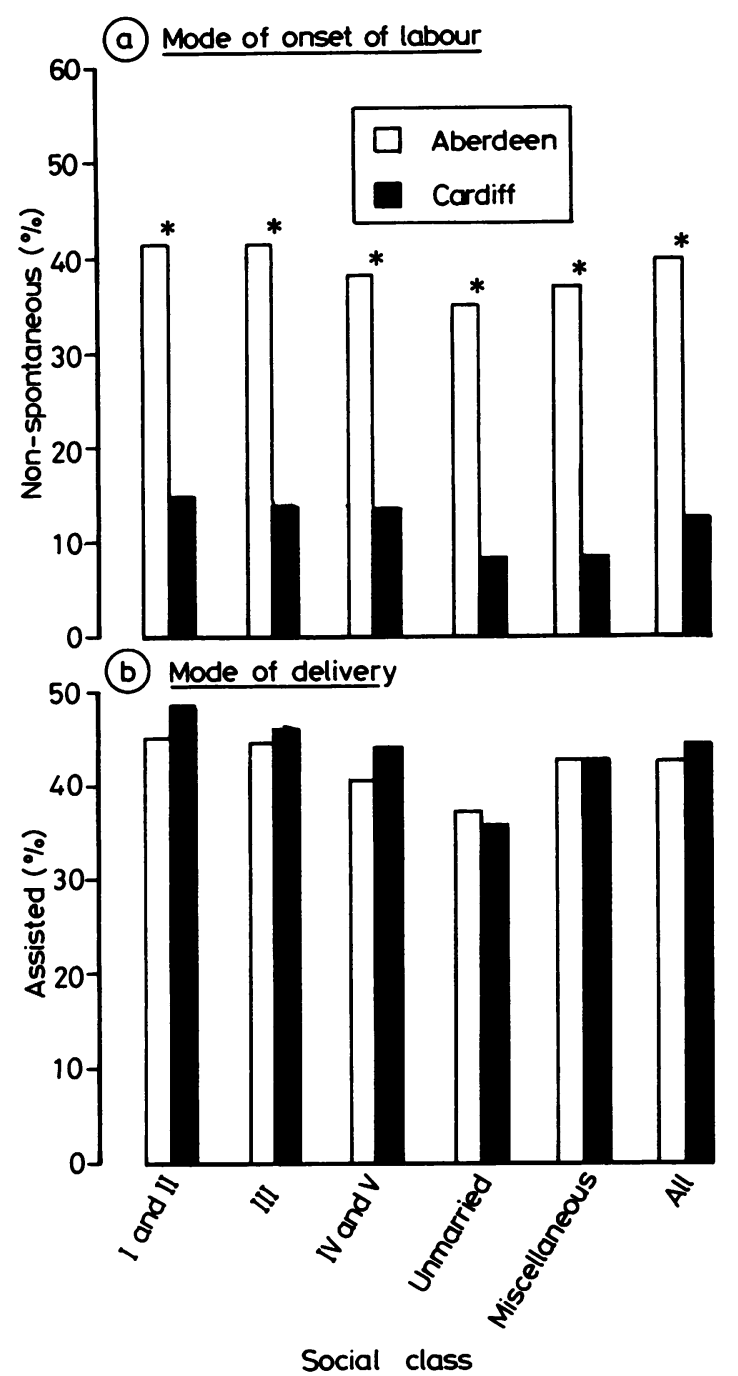

Fig 2

this relationship for teenage pregnancies and smoking only. In both cases the relative risk is higher in the CBS than the AMNDB. These differences are almost totally due to an increased likelihood of teenage pregnancies or smoking in social classes I and II in the AMNDB compared with the CBS.

Obstetric practice (fig 2 and table 7)

Overall, there are no significant social class differences in the rates of induction or assisted delivery. This pattern is replicated in both areas.

Outcomes (fig 3 and table 8)

Again, the social class relationships go in the expected directions, lower class mothers being more likely to
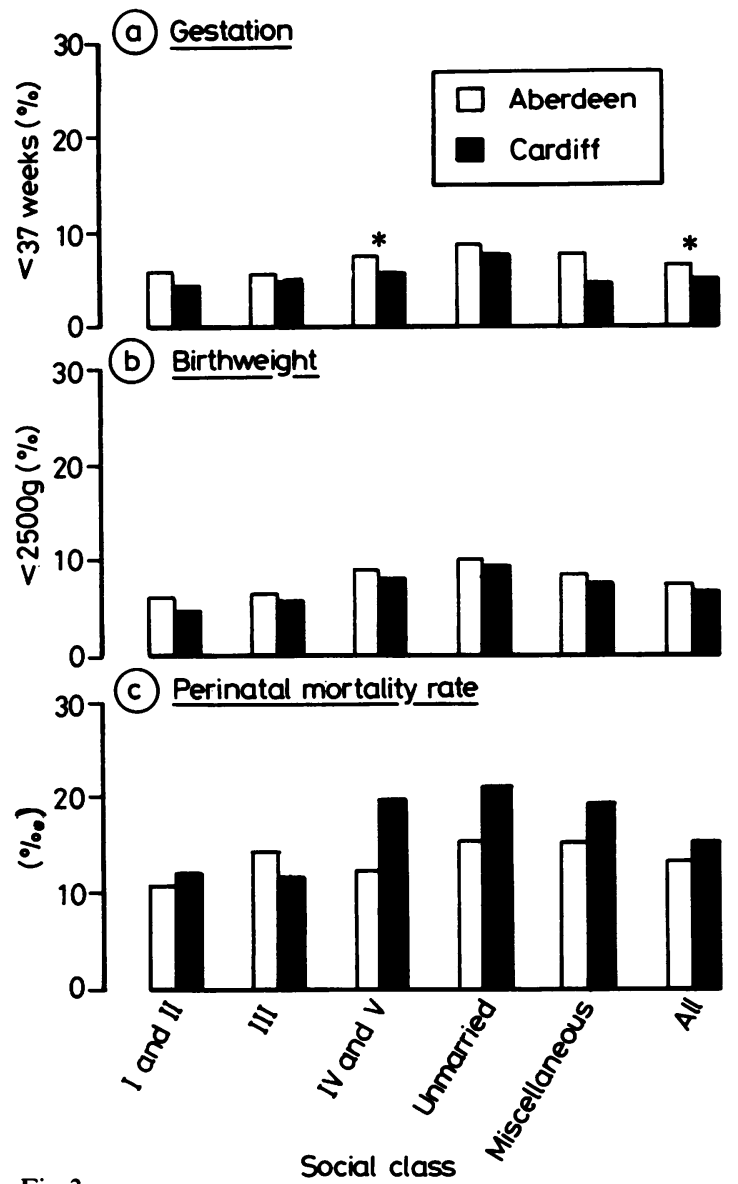

Fig 3

deliver preterm and have smaller babies. Although there is a raised relative risk of perinatal death for classes IV and V, this does not reach statistical significance. These social class differences do not vary significantly between the areas.

In summary, social class gradients are as expected. In general the size of the relative risk does not vary significantly between locations.

\section{Discussion}

The intention of this paper was primarily to provide a descriptive contribution to the literature on regional and social class variations in perinatal outcome. National perinatal mortality statistics have repeatedly shown large differences in mortality in different areas of the country and by social class. Because of the way in which such statistics are gathered and reported and because of the focus on perinatal death, studies of area differences have tended to be based on large areas. We 
Table 5 Area comparison: outcomes

\begin{tabular}{|c|c|c|c|c|c|c|c|c|c|c|c|c|}
\hline \multirow[b]{3}{*}{$\mathbf{N}$} & \multicolumn{12}{|c|}{ Social class } \\
\hline & \multicolumn{2}{|l|}{ I \& II } & \multicolumn{2}{|l|}{ III } & \multicolumn{2}{|c|}{ IV \& V } & \multicolumn{2}{|c|}{ Unmarried } & \multicolumn{2}{|c|}{ Miscellaneous } & \multicolumn{2}{|l|}{$A l l$} \\
\hline & $\begin{array}{l}\text { A } \\
907\end{array}$ & $\begin{array}{l}\mathrm{C} \\
2296\end{array}$ & $\begin{array}{l}A \\
1853\end{array}$ & $\begin{array}{l}\text { C } \\
4755\end{array}$ & $\begin{array}{l}\mathrm{A} \\
1127\end{array}$ & $\begin{array}{l}\text { C } \\
1539\end{array}$ & $\begin{array}{l}A \\
910\end{array}$ & $\begin{array}{l}C \\
2441\end{array}$ & $\begin{array}{l}\text { A } \\
192\end{array}$ & $\begin{array}{l}C \\
862\end{array}$ & $\begin{array}{l}\text { A } \\
4989\end{array}$ & $\begin{array}{l}C \\
11893\end{array}$ \\
\hline $\begin{array}{l}<37 \text { weeks } \\
\% \\
\text { RR } \\
95 \% \text { CI }\end{array}$ & $\begin{array}{r}49 \\
5 \cdot 4 \\
0\end{array}$ & $\begin{array}{l}\quad 95 \\
\quad 4 \cdot 1 \\
-31 \\
-1 \cdot 83\end{array}$ & $\begin{array}{r}109 \\
5.9 \\
1.0\end{array}$ & $\begin{array}{c}223 \\
25^{*} \\
-1 \cdot 7\end{array}$ & $\begin{array}{r}92 \\
8.2 \\
1 \\
1.0\end{array}$ & $\begin{array}{c}87 \\
4 \cdot 7 \\
-1 \cdot 91\end{array}$ & $\begin{array}{r}84 \\
9 \cdot 2 \\
\\
0\end{array}$ & $\begin{array}{l}195 \\
8.0 \\
.16 \\
-1.48\end{array}$ & \multicolumn{2}{|c|}{$\begin{array}{c}1.73 \\
0.97-3.06\end{array}$} & $\begin{array}{c}1 \cdot 29^{*} \\
1 \cdot 14-1 \cdot 47\end{array}$ & $\begin{array}{c}639 \\
.29 * 5 \\
4-1.47\end{array}$ \\
\hline $\begin{array}{l}<2500 \mathrm{~g} \\
\% \\
\text { RR } \\
95 \% \text { CI }\end{array}$ & \multicolumn{2}{|c|}{$\begin{array}{c}1.31 \\
0.96-1.80\end{array}$} & $\begin{array}{c}1.06 \\
0.88-1.29\end{array}$ & $\begin{array}{c}323 \\
6 \cdot 8 \\
.06 \\
-1 \cdot 29\end{array}$ & $\begin{array}{r}113 \\
10.0 \\
0.9\end{array}$ & $\begin{array}{c}135 \\
8.8 \\
\cdot 14^{8.8} \\
-1.45\end{array}$ & $\begin{array}{l}97 \\
10.7 \\
\\
0 .\end{array}$ & $\begin{array}{l}242 \\
.08 \\
-1 \cdot 94\end{array}$ & $\begin{array}{l}16 \\
8.3 \\
\\
0 .\end{array}$ & $\begin{array}{c}66 \\
7.7 \\
.09 \\
-1.84\end{array}$ & $\begin{array}{r}414 \\
8 \cdot 3 \\
\\
0.9\end{array}$ & $\begin{array}{r}\quad 872 \\
\quad 7 \cdot 3 \\
1 \cdot 11 \\
9-1 \cdot 25\end{array}$ \\
\hline $\begin{array}{l}\text { PND } \\
\text { Per } 1000 \\
\text { PR } \\
95 \% \text { CI }\end{array}$ & $\begin{array}{l}10 \\
11.0 \\
\\
0\end{array}$ & $\begin{array}{c}29 \\
12 \cdot 6 \\
.87 \\
-1 \cdot 78\end{array}$ & 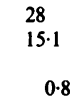 & \begin{tabular}{l}
\multicolumn{1}{c}{56} \\
$11 \cdot 8$ \\
.28 \\
-2.01
\end{tabular} & $\begin{array}{l}14 \\
12 \cdot 4 \\
0.3\end{array}$ & $\begin{array}{l}32 \\
20 \cdot 8 \\
.60 \\
-1 \cdot 11\end{array}$ & $\begin{array}{l}14 \\
15 \cdot 4 \\
\\
0 .\end{array}$ & $\begin{array}{l}{ }^{54} \\
22 \cdot 1 \\
-70^{2} \cdot 1 \cdot 24\end{array}$ & $\begin{array}{c}0.79 \\
0.24-2.67\end{array}$ & $\begin{array}{l}17 \\
19 \cdot 7 \\
.79 \\
-2 \cdot 67\end{array}$ & $\begin{array}{c}0.87 \\
0.66-1 \cdot 14\end{array}$ & $\begin{array}{c}188 \\
15 \cdot 8 \\
6 \cdot 87 \\
6-1 \cdot 14\end{array}$ \\
\hline
\end{tabular}

$* \mathrm{p}<0.05$

Table 6 Social class gradients: obstetric population

\begin{tabular}{|c|c|c|c|}
\hline \multirow[b]{2}{*}{$\mathbf{N}$} & Aberdeen & Cardiff & \multirow[t]{2}{*}{ Overall } \\
\hline & $\begin{array}{l}\text { IV \& V I \& II } \\
1127\end{array}$ & $\begin{array}{ll}\text { IV \& V } & \text { I\& II } \\
1539 & 2296\end{array}$ & \\
\hline $\begin{array}{l}\leqslant 154 \mathrm{~cm} \\
\%\end{array}$ & $\begin{array}{cc}278 & 130 \\
24 \cdot 7 & 14 \cdot 3\end{array}$ & $\begin{array}{cc}301 & 252 \\
19.6 & 11.0\end{array}$ & \\
\hline $\begin{array}{l}\text { RR } \\
95 \% \mathrm{CI}\end{array}$ & $\begin{array}{c}1.72^{*} \\
1.43-2.07\end{array}$ & $\begin{array}{c}1.78^{*} \\
1.53-2.08\end{array}$ & $\begin{array}{l}1.8^{*} \\
1.6-2.0\end{array}$ \\
\hline $\begin{array}{l}<20 \text { years } \\
\%\end{array}$ & $\begin{array}{cc}302 & 42 \\
26.8 & 4.6\end{array}$ & $\begin{array}{cc}457 & 43 \\
29.7 & 1.9\end{array}$ & \\
\hline $\begin{array}{l}\text { RR } \\
\mathbf{9 5 \%} \text { CI }\end{array}$ & $\begin{array}{c}5.79 * \\
4.46-7.50\end{array}$ & $\begin{array}{c}15 \cdot 86^{*} \\
12 \cdot 78-19 \cdot 68\end{array}$ & $\begin{array}{l}10 \cdot 1^{*} \\
8 \cdot 6-11 \cdot 9\end{array}$ \\
\hline $\begin{array}{l}>29 \text { years } \\
\%\end{array}$ & $\begin{array}{cl}57 & 160 \\
5.1 & 17.6\end{array}$ & $\begin{array}{cc}88 & 549 \\
5.7 & 23.9\end{array}$ & \\
\hline $\begin{array}{l}\text { RR } \\
95 \% \text { CI }\end{array}$ & $\begin{array}{c}0.29^{*} \\
0.22-0.38\end{array}$ & $\begin{array}{c}0.24^{*} \\
0.20-0.29\end{array}$ & $\begin{array}{c}0.3^{*} \\
0.2-0.3\end{array}$ \\
\hline $\begin{array}{l}\text { Smokers } \\
\%\end{array}$ & $\begin{array}{cc}624 & 254 \\
55.4 & 28.0\end{array}$ & $\begin{array}{cc}727 & 422 \\
47 \cdot 2 & 18.4\end{array}$ & \\
\hline $\begin{array}{l}\text { RR } \\
95 \% \text { CI }\end{array}$ & $\begin{array}{c}1.98^{*} \\
1.78-2.20\end{array}$ & $\begin{array}{c}2.57^{*} \\
2.33-2.83\end{array}$ & $\begin{array}{c}2 \cdot 3 * \\
2 \cdot 1-2 \cdot 5\end{array}$ \\
\hline
\end{tabular}

* $p<0.05$

have made use of the opportunity provided by the comparable data bases of the CBS and the AMNDB to examine smaller regions and factors which national studies have shown to be associated with perinatal mortality. This study has confirmed the well-known existence of such social class and area differences.

Moreover, we have demonstrated that, for these two regions and in respect of the variables considered here, the social class differences are similar in the CBS and AMNBD and the area differences are relatively consistent for each of the social classes. In this sense,
Table 7 Social class gradients: obstetric practice

\begin{tabular}{|c|c|c|c|}
\hline \multirow[b]{2}{*}{$\mathbf{N}$} & Aberdeen & Cardiff & \multirow[t]{2}{*}{ Overall } \\
\hline & $\begin{array}{l}\text { IV \& V I \& II } \\
1127 \quad 907\end{array}$ & $\begin{array}{l}\text { IV \& V I \& II } \\
1539\end{array} 2296$ & \\
\hline $\begin{array}{l}\text { Induction } \\
\%\end{array}$ & $\begin{array}{cc}450 & 388 \\
39 \cdot 2 & 42 \cdot 8\end{array}$ & $\begin{array}{cc}207 & 357 \\
13.5 & 15.6\end{array}$ & \\
\hline $\begin{array}{l}\text { RR } \\
95 \% \text { CI }\end{array}$ & $\begin{array}{c}0.93 \\
0.84-1.04\end{array}$ & $\begin{array}{c}0.87 \\
0.74-1.01\end{array}$ & $\begin{array}{c}0.90 \\
0.83-1.00\end{array}$ \\
\hline $\begin{array}{l}\text { Assisted } \\
\text { delivery } \\
\%\end{array}$ & $\begin{array}{cc}471 & 424 \\
41 \cdot 8 & 46 \cdot 7\end{array}$ & $\begin{array}{cc}696 & 1137 \\
45.2 & 49.5\end{array}$ & \\
\hline $\begin{array}{l}\text { RR } \\
95 \% \text { CI }\end{array}$ & $\begin{array}{c}0.89 \\
0.81-1.00\end{array}$ & $\begin{array}{c}0.91 \\
0.85-1.00\end{array}$ & $\begin{array}{c}0.91 \\
0.86-1.00\end{array}$ \\
\hline
\end{tabular}

Table 8 Social class gradients: outcome

\begin{tabular}{|c|c|c|c|}
\hline & Aberdeen & Cardiff & Overall \\
\hline $\mathbf{N}$ & $\begin{array}{l}\text { IV \& V I \& II } \\
1127 \quad 907\end{array}$ & $\begin{array}{l}\text { IV \& V I \& II } \\
1539\end{array}$ & \\
\hline $\begin{array}{l}\text { Preterm } \\
\text { delivery } \\
\% \\
\text { RR } \\
95 \% \text { CI }\end{array}$ & $\begin{array}{cc}92 & 49 \\
8 \cdot 2 & 5 \cdot 4 \\
1 \cdot 51^{*} & \\
1 \cdot 08-2 \cdot 11\end{array}$ & $\begin{array}{cc}87 & 95 \\
5 \cdot 7 & 4 \cdot 1 \\
1 \cdot 37 * & \\
1 \cdot 03-1 \cdot 81\end{array}$ & $\begin{array}{c}1.4^{*} \\
1 \cdot 2-1 \cdot 8\end{array}$ \\
\hline $\begin{array}{l}\text { LBWT } \\
\% \\
\text { RR } \\
95 \% \text { CI }\end{array}$ & $\begin{array}{cc}113 & 55 \\
10 \cdot 0 & 6 \cdot 1 \\
1.65^{*} & \\
1.22-2.24\end{array}$ & $\begin{array}{cc}135 & 106 \\
8.8 \quad 4.6 \\
1.90^{*} \\
1.49-2.42\end{array}$ & $\begin{array}{l}1.8^{*} \\
1.5-2.2\end{array}$ \\
\hline $\begin{array}{l}\text { PND } \\
\text { Per } 1000 \\
\text { RR } \\
95 \% \text { CI }\end{array}$ & $\begin{array}{cl}14 \quad 10 \\
12 \cdot 4 \quad 11 \cdot 0 \\
1 \cdot 13 \\
0.50-2.52\end{array}$ & $\begin{array}{ll}32 & 29 \\
20.8 & 12.6 \\
1.65 \\
1.00-2 \cdot 69\end{array}$ & $\begin{array}{c}1.48 \\
0.97-2 \cdot 25\end{array}$ \\
\hline
\end{tabular}


social class and area may be seen as independently associated with these variables.

These findings for perinatal mortality and associated factors correspond to those reported for infant and adult mortality in the Decennial Supplement ${ }^{2}$ for larger areas.

$A$ priori, the independence of area and social class "effects" is surprising. As Illsley has pointed out, ${ }^{7}$ it would seem reasonable to expect that, for example, the living conditions and styles of life of social classes I and II would vary less by region than those of social classes IV and V. Similarly, because of the occupational basis of the social class classification, it might be expected that the "effects" of social class would vary according to the different local occupational and industrial systems - although, clearly, such an expectation would be modified by the 'indirect' classification of women according to their husbands' occupations. ${ }^{1}$

Area and social class differences are not amenable to simple or unambiguous interpretation. Classification by area may indicate factors as diverse as environmental pollution, resource allocation, and cultural milieux. As this paper has shown, in the case of induction, area differences also indicate differences in obstetric practice. In the present study, the comparison has been made between Scottish and Welsh areas, between oil-rich Aberdeen and South Glamorgan with its higher than average unemployment. Whether social class as defined here has any sociological or economic meaning is unclear. Furthermore, the classification of women by their husbands' occupations adds to the problems of interpretation. $^{8}$ However, empirically, this classification has consistently been shown to be a good discriminator of perinatal outcome.

Social class differences may reflect relative prosperity and differences in the material conditions of life. As this paper has shown in the cases of smoking and the age at which family building starts, social class differences may also reflect different patterns of behaviour. It is interesting to note that it is in these 'behavioural' variables that there is some evidence that area and social class differences interact in such a way that area differences are greater for people in the 'higher' than in the 'lower' social classes.

Because of the difficulties associated with the interpretation of area and social class differences, the implications of their independent association with the variables considered here are obscure. All that this study has been able to suggest is that, whatever the relevant characteristics of social class are, their net effects are similar in Aberdeen and Cardiff and that, whatever the relevant characteristics of the areas are, their net effects are similar for the different social classes. Caution must be exercised in any extrapolation of the results from this perhaps untypical comparison to comparisons between other areas in the United Kingdom.

The strategy of the comparative study of social class patterns in obstetric outcomes in relatively small areas brings difficulties with it. One such difficulty is illustrated by the findings reported in this paper. AMNDB mothers are more likely than those in the CBS to be of short stature and to smoke. Consistent with this pattern, babies born in Aberdeen were significantly more likely to weigh $2500 \mathrm{~g}$ or less. However, in terms of perinatal deaths, the mortality rates in Aberdeen are similar to those in Cardiff, if anything, being slightly lower.

It may be that these figures reflect real differences in the association between the risk factors and perinatal death in the two locations. Even over a six year period, however, the numbers of deaths occurring in Cardiff and Aberdeen are insufficient for any meaningful comparison of the social class patterns in mortality to be made. Although the strategy of the comparative study of social class patterns in relatively small areas has much to recommend it, it will always be the case that such studies will be, at best, suggestive of the factors that contribute to the social patterning of relatively infrequent outcomes.

We are grateful to Iain Chalmers, Alison Macfarlane, Sally McIntyre, Robert Newcombe, and other colleagues both within the National Perinatal Epidemiology Unit, the University of Wales Department of Medical Computing and Statistics, and the MRC Medical Sociology Unit, and outside, for their constructive criticisms. Thanks also to Jini Hetherington, Lesley Mierh, and Janelle Rogers for typing numerous drafts. At the time the research was conducted, Diana Elbourne was supported by the DHSS and Colin Pritchard by the MRC. The Cardiff Births Survey is funded by the Welsh Office and the South Glamorgan Health Authority.

\section{References}

${ }^{1}$ See for example, Macfarlane A, Mugford M. Birth counts: Statistics of pregnancy and childbirth, London: HMSO, 1984.

2 Office of Population Censuses and Surveys. Occupational mortality. The Registrar General Decennial Supplement for England and Wales 1970-1972 Series DS no 1, London: HMSO 1978 p182.

${ }^{3}$ Samphier ML, Thompson B. The Aberdeen maternity and neonatal data bank, in Mednick SA, Baert AE, eds. Prospective longitudinal research London: OUP, 1981. 
${ }^{4}$ Andrews J, Davies K, Chalmers I, Campbell H. The Cardiff Births Survey: development, perinatal mortality, birthweight and length of gestation. In: Harper PS, Sunderland E, eds. Genetic and population studies in Wales. Cardiff: University of Wales Press (in press).

${ }^{5}$ Mantel N, Haenszel W. Statistical aspects of the analysis of data from retrospective studies of disease. J Natl Cancer Inst, 1959; 22: 719-48.
${ }^{6}$ Miettinen OS. Estimability and estimation in case-referent studies. AM J Epidemiol, 1976; 103: 226-35.

${ }^{7}$ Illsley R. Professional or public health? Sociology in Health and Medicine. London: Nuffield Provincial Hospitals Trust, 1980, 16.

${ }^{8}$ Macfarlane A. Official statistics and women's health and illness, EOC Research Bulletin no 4, Autumn 1980: 43-77. 